\title{
Reflection and Transmission Coefficients of Linearly Polarized Light Passing Through Three Separated Plane-Parallel Plates in Vacuum
}

\author{
Ymer Halimi, Besim Xhafa and Albert Januzaj \\ Faculty of Medicine, University of Prishtina, Prishtina, Kosovo
}

\begin{abstract}
State of polarization of light on reflection and transmission is a subject of change. This change can be determined by determining the coefficients of reflection and transmission. When coefficients are complex numbers, we obtain an ellyptic polarization. This phenomenon appears also when the incident light is linearly or circularly polarized. In this study, the problem of three equally separated plates in vacuum is discussed. The incident light is considered linearly polarized and with a high degree of coherence. Interference on reflection and absorption are not taken into consideration. For determination of coefficients $r$ and $t$, the recursive Wolter's formulas are used. Formulas for $\mathrm{r}$ and $\mathrm{t}$ obtained for isotropic plates are transformed for anisotropic plates for special directions of optical axes of crystalline plates. The accuracy of obtained formulas is verified for the case of a single plate.
\end{abstract}

Key words: Light, polarization, reflection, transmission, coefficients, plates

\section{INTRODUCTION}

State of polarization of light on reflection and transmission is a subject of change (Zander et al., 1983). In order to determine the state of polarized light, it is necessary to determine the coefficients of reflection $r$ and transmission t. Coefficients are complex numbers, with values not $>1$. In other words, reflection coefficient has the form:

$$
\Upsilon=\frac{P+i Q}{K+i L}
$$

Whereas, the transmission coefficient, the form:

$$
\mathrm{t}=\frac{1}{\mathrm{~K}+\mathrm{iL}}
$$

In Eq. 2, we take: $P=1$ and $Q=0$. In order to determine the coefficients $r$ and $t$, first we must determine the parameters $\mathrm{P}, \mathrm{Q}, \mathrm{K}$ and $\mathrm{L}$. In the case of only one boundary surface, coefficients $r$ and $t$ are determined by Fresnel's formulas.

In this case, we have to deal with coherent and linearly polarized incident light. Incident light has two components: $\mathrm{E}_{\mathrm{n}}$, oscillating perpendicularly to the incident plane and $\mathrm{E}_{\mathrm{p}}$ oscillating parallel to the incident plane. Therefore, these components of light waves, on reflection and transmission are expressed by equations:

$$
E_{p}=\Upsilon_{p} E_{o p}^{\prime} E_{n}=\Upsilon_{n} E_{o n}
$$

and;

$$
E_{p}=t_{p} E_{o p}, E_{n}=t_{n} E_{o n}
$$

Where, $\mathrm{E}_{0}$ is the incident light. From the Fresnel's equation (Born and Wolf, 1999) for the coefficient $r$ for this case, we have:

$$
\begin{gathered}
\mathrm{r}_{\mathrm{p}}=\frac{\mathrm{n}_{1} \cos \beta_{2}-\mathrm{n}_{2} \cos \beta_{1}}{\mathrm{n}_{1} \cos \beta_{2}+\mathrm{n}_{2} \cos \beta_{1}} \\
\mathrm{r}_{\mathrm{n}}=\frac{\mathrm{n}_{2} \cos \beta_{2}-\mathrm{n}_{1} \cos \beta_{1}}{\mathrm{n}_{2} \cos \beta_{2}+\mathrm{n}_{1} \cos \beta_{1}}
\end{gathered}
$$

We divide both side of Eq. 5 by $n_{1} . n_{2}$ and obtain the ratios with the same subscripts:

$$
\mathrm{r}_{\mathrm{p}}=\frac{\frac{\cos \beta_{2}}{\mathrm{n}_{2}}-\frac{\cos \beta_{1}}{\mathrm{n}_{1}}}{\frac{\cos \beta_{2}}{\mathrm{n}_{2}}+\frac{\cos \beta_{1}}{\mathrm{n}_{1}}}
$$

In order to simplify the operations further, we take the substitution (Moser, 1985 ):

$$
g=\left[\begin{array}{l}
g p \\
g n
\end{array}\right]=\left[\begin{array}{c}
\frac{\cos \beta}{n} \\
n \cos \beta
\end{array}\right]
$$

Finally, equations for $\mathrm{r}_{\mathrm{p}}$ and $\mathrm{r}_{\mathrm{n}}$ have the same terms: 


$$
\mathrm{r}=\frac{\mathrm{g}_{2}-\mathrm{g}_{1}}{\mathrm{~g}_{2}+\mathrm{g}_{1}}
$$

Similarly, for the coefficient $t$, we have:

$$
\mathrm{t}=\frac{2 \mathrm{~g}_{2}}{\mathrm{~g}_{2}+\mathrm{g}_{1}}
$$

In cases when we have $>1$ plate or more than one boundary surface, respectively, the coefficient $r$ is determined by recursive Walter's formulas:

$$
\mathrm{Y}_{\mathrm{j}}=\frac{\mathrm{B}_{\mathrm{j}}}{\mathrm{N}_{\mathrm{j}}}
$$

And similarly, for the coefficient t:

$$
t_{j}=\frac{2^{j} \prod_{i=0}^{j-1} g_{i}}{N_{j}}
$$

Where $\mathrm{j}$ indicates boundary surfaces which in this case for three isotropic plates is $\mathrm{j}=6$. On the other hand, $\mathrm{B}_{\mathrm{j}}$ and $\mathrm{N}_{\mathrm{j}}$ are determined by:

$$
\begin{aligned}
B_{j}= & {\left[\left(g_{j}-g_{j-1}\right) N_{j-1}+\left(g_{j}+g_{j-1}\right) B_{j-1}\right] \cos \varphi_{j-1}+} \\
& i\left[\left(g_{j}-g_{j-1}\right) N_{j-1}-\left(g_{j}+g_{j-1}\right) B_{j-1}\right] \sin \varphi_{j-1} \\
N_{j}= & {\left[\left(g_{j}-g_{j-1}\right) N_{j-1}+\left(g_{j}+g_{j-1}\right) B_{j-1}\right] \cos \varphi_{j-1}+} \\
& i\left[\left(g_{j}+g_{j-1}\right) N_{j-1}-\left(g_{j}-g_{j-1}\right) B_{j-1}\right] \sin \varphi_{j-1}
\end{aligned}
$$

When $\mathrm{j}=1$, we obtain the Fresnel's formulas for one boundary surface. In Eq. 13, 14 for B and $N$ the phase $\varphi$ appears, due to the transmission of light through the plates and vacuum spaces. The equation for the phase is (Moser, 1985):

$$
\varphi_{j}=\frac{2 \pi}{\lambda} d_{j} n_{j} \cos \beta_{j}
$$

Where:

$\lambda=$ The wavelength of incident light

$\mathrm{d}_{\mathrm{j}}=$ The thickness of the plate which in this case is the same for spacing in vacuum

$n_{j}=$ The index of refraction of the $j$ th plate

$\beta_{j}=$ The angle of refraction of light on the exit of the plates

According to the Eq. 16 for the angle of refraction:

$$
\sin \beta=\frac{\sin \alpha}{n}
$$

The phase angle is written in the form:

$$
\varphi \mathrm{j}=\frac{2 \pi}{\lambda} \mathrm{d}_{\mathrm{j}} \sqrt{\mathrm{n}_{\mathrm{j}}^{2}-\sin ^{2} \alpha}
$$

Where $\alpha$ is the angle which incident light covers with the normal $\mathrm{n}$ which is given and therefore in the Eq. 17, the angle of refraction is eliminated.

\section{MATERIALS AND METHODS}

The $r$ and $t$ coefficients for an isotropic plan-parallel plate for a linearly polarized incident light: Before determination of $\mathrm{B}$ and $\mathrm{N}$ for the order $\mathrm{j}=6$ for three plates, let we first determine them for two surfaces that is for one plate, respectively (Fig. 1). Surfaces of the plate are parallel and placed in vacuum. For the boundary surface vacuum-plate, we take the notation $g_{0}$ and $g$ for the boundary surface plate-vacuum. In Eq. 13 and 14, we substitute the values for $\mathrm{j}=2$ and respective $\mathrm{g}-\mathrm{s}$ and determine $\mathrm{B}_{2}$ and $\mathrm{N}_{2}$, the numerator and the denominator of reflection and transmission coefficients, respectively:

$$
\begin{aligned}
\mathrm{B}_{2}= & {\left[\left(g_{0}-g\right)\left(g+g_{0}\right)+\left(g_{0}+g\right)\left(g-g_{0}\right)\right] \sin \varphi+} \\
& i\left[\left(g_{0}-g\right)\left(g+g_{0}\right)-\left(g_{0}-g\right)\left(g+g_{0}\right)\right] \sin \varphi \\
N_{2}= & {\left[\left(g_{0}+g\right)\left(g+g_{0}\right)+\left(g_{0}-g\right)\left(g-g_{0}\right)\right] \cos \varphi+} \\
& i\left[\left(g_{0}-g\right)\left(g+g_{0}\right)-\left(g_{0}-g\right)\left(g-g_{0}\right)\right] \sin \varphi
\end{aligned}
$$

Finally, for $\mathrm{B}_{2}$ and $\mathrm{N}_{2}$, we obtain:

$$
\begin{gathered}
\mathrm{B}_{2}=2 \mathrm{i}\left(\mathrm{g}_{0}^{2}-\mathrm{g}^{2}\right) \sin \varphi \\
\mathrm{N}_{2}=4 \mathrm{~g}_{0} \mathrm{~g} \cos \varphi+2 \mathrm{i}\left(\mathrm{g}_{0}^{2}-\mathrm{g}^{2}\right) \sin \varphi
\end{gathered}
$$

For the reflection coefficient for the surface $j=2$, we obtain the equation of the form:

$$
\mathrm{r}_{2}=\frac{i 0.5\left(\frac{\mathrm{g}_{0}}{\mathrm{~g}}-\frac{\mathrm{g}}{\mathrm{g}_{0}}\right) \sin \varphi}{\cos \varphi+i 0.5\left(\frac{\mathrm{g}_{0}}{\mathrm{~g}}+\frac{\mathrm{g}}{\mathrm{g}_{0}}\right) \sin \varphi}
$$

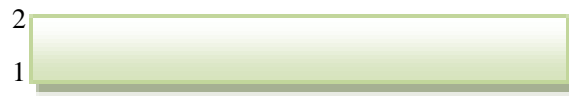

Fig. 1: Surface of one plate 
Then we make the substitutions:

$$
\mathrm{R}=0.5\left(\frac{\mathrm{g}_{0}}{\mathrm{~g}}+\frac{\mathrm{g}}{\mathrm{g}_{0}}\right)
$$

and;

$$
\mathrm{R}^{\prime}=0.5\left(\frac{\mathrm{g}_{0}}{\mathrm{~g}}-\frac{\mathrm{g}}{\mathrm{g}_{0}}\right)
$$

Finally, for the coefficient $\mathrm{r}_{2}$, we obtain:

$$
\mathrm{r}_{2}=\frac{\mathrm{i} \mathrm{R}^{\prime} \sin \varphi}{\cos \varphi+\mathrm{iR} \sin \varphi}
$$

and for the coefficient $t_{2}$ :

$$
\mathrm{t}_{2}=\frac{1}{\cos \varphi+\mathrm{iR} \sin \varphi}
$$

We substitute the coefficients $r$ and $t$ in the Eq. 3 and 4 for the parallel and normal components $\left(\mathrm{E}_{\mathrm{p}, \mathrm{n}}\right)$. Thus for the case $j=2$, we have determined the parameters of Eq. 1 and 2 which have the form (Moser et al.,1987):

$$
\begin{gathered}
P_{p, n}=0 \quad Q_{p, n}=R_{p, n}^{\prime} \sin \varphi \\
K_{p, n}=\cos \varphi \quad L_{p, n}=R_{p, n} \sin \varphi
\end{gathered}
$$

Having determined $r$ and $t$ coefficients for a single plate, we proceed for a case of three separated planeparallel plates (Fig. 2). Thickness and distance between plates is taken to be $1 \mathrm{~mm}$.

Determination of numerator $B_{j}$ in $\mathbf{E q} .11$ for $\mathbf{j}=6$ : Now we will determine the parameters $P, Q, K$ and $L$ for $j=6$. For this purpose, we start from the Eq. 13 and 14 finding first $\mathrm{B}_{3}, \mathrm{~N}_{3}, \mathrm{~B}_{4}, \mathrm{~N}_{4}, \mathrm{~B}_{5}, \mathrm{~N}_{5}$ and $\mathrm{B}_{6}, \mathrm{~N}_{6}$. Meanwhile, $\mathrm{B}_{2}$ and $\mathrm{N}_{2}$ are determined by the Eq. 18 and 19, respectively:

$$
\begin{gathered}
B_{3}=\left(g-g_{0}\right) N_{2}\left(\cos \varphi_{0}+i \sin \varphi_{0}\right)+ \\
\left(g+g_{0}\right) B_{2}\left(\cos \varphi_{0}-i \sin \varphi_{0}\right)
\end{gathered}
$$

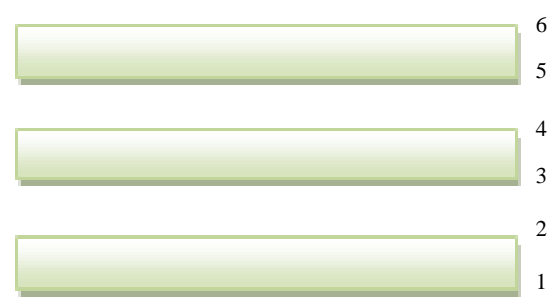

Fig. 2: Three separated plane parall plates

$$
\begin{aligned}
\mathrm{N}_{3}= & \left(g+g_{0}\right) N_{2}\left(\cos \varphi_{0}+i \sin \varphi_{0}\right)+ \\
& \left(g-g_{0}\right) B_{2}\left(\cos \varphi_{0}-i \sin \varphi_{0}\right) \\
B_{4}= & (g-g) N_{3}(\cos \varphi+i \sin \varphi)+ \\
& \left(g_{0}+g\right) B_{3}(\cos \varphi-i \sin \varphi) \\
N_{4}= & \left(g_{0}+g\right) N_{3}(\cos \varphi+i \sin \varphi)+ \\
& \left(g_{0}-g\right) B_{3}(\cos \varphi-i \sin \varphi) \\
B_{5}= & \left(g-g_{0}\right) N_{4}\left(\cos \varphi_{0}+i \sin \varphi_{0}\right)+ \\
& \left(g+g_{0}\right) B_{4}\left(\cos \varphi_{0}-i \sin \varphi_{0}\right) \\
N_{5}= & \left(g+g_{0}\right) N_{4}\left(\cos \varphi_{0}+i \sin \varphi_{0}\right)+ \\
& \left(g-g_{0}\right) B_{4}\left(\cos \varphi_{0}-i \sin \varphi_{0}\right)
\end{aligned}
$$

And for the last surface we have:

$$
\begin{aligned}
\mathrm{B}_{6}= & \left(\mathrm{g}_{0}+\mathrm{g}\right) \mathrm{N}_{5}(\cos \varphi+\mathrm{i} \sin \varphi)+ \\
& \left(\mathrm{g}_{0}+\mathrm{g}\right) \mathrm{N}_{5}(\cos \varphi-i \sin \varphi) \\
\mathrm{N}_{6}= & \left(g_{0}+\mathrm{g}\right) \mathrm{N}_{5}(\cos \varphi+i \sin \varphi)+ \\
& \left(g_{0}-g\right) \mathrm{B}_{5}(\cos \varphi-i \sin \varphi)
\end{aligned}
$$

Now, substituting in successive manner Eq. 25-32 in Eq. 31 and after some mathematical operations, we obtain:

$$
\mathrm{g}_{0}^{2}+\mathrm{g}^{\uparrow}
$$

Now, we take the real part of Eq. 33 which corresponds to $\mathrm{P}_{6}$ and the imaginary part of Eq. 33 which corresponds to $\mathrm{Q}_{6}$.

\section{Determination of $P_{6}$ and $Q_{6}$ parameters:}

$$
\begin{aligned}
& \mathrm{P}_{\downarrow} 6=\operatorname{Re} \mathrm{B}_{\downarrow} 6=-32 \mathrm{~g}_{\downarrow} \mathrm{o}^{\uparrow} 2 \mathrm{~g}^{\uparrow} 2\left(\mathrm{~g}_{\downarrow} \mathrm{o}^{\uparrow} 2-\mathrm{g}^{\uparrow} 2\right) \cos ^{\uparrow} 2 \varphi \sin \varphi \cos \varphi_{\downarrow} \circ \sin \varphi_{\downarrow} \mathrm{o}+32 \mathrm{~g}_{\downarrow} \mathrm{o}^{\uparrow} 2 \mathrm{~g}^{\uparrow} 2
\end{aligned}
$$

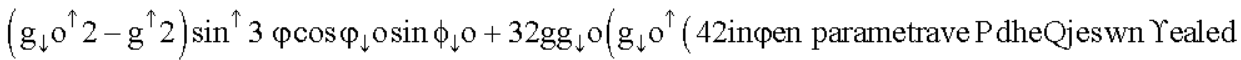




$$
\begin{aligned}
\mathrm{P}_{\downarrow} \sigma= & 32 \mathrm{~g}_{\downarrow} \mathrm{o}^{\uparrow} 2 \mathrm{~g}^{\uparrow} 2\left(\mathrm{~g}_{\downarrow} \mathrm{o}^{\uparrow} 2-\mathrm{g}^{\uparrow} 2\right)\left(-\cos ^{\uparrow} 2 \varphi \sin \varphi \cos \varphi_{\downarrow} \mathrm{o} \sin \varphi_{\downarrow} O+\sin ^{\uparrow} 3 \varphi \cos \varphi_{\downarrow} O \sin \varphi_{\downarrow} 0-\right. \\
& \cos ^{\uparrow} 2 \varphi \sin \varphi \cos \varphi_{\downarrow} O \sin \varphi_{\downarrow} O-0.5 \sin ^{\uparrow} 3 \varphi \cos \varphi_{\downarrow} O \sin \varphi_{\downarrow} O+2 \cos _{\uparrow} 2 \varphi \sin \varphi \cos \varphi
\end{aligned}
$$

or finally;

$$
\mathrm{P}_{6}=0
$$

Whereas, the imaginary part of the Eq. 26 that corresponds to $Q_{6}$ is:

$\mathrm{Q}_{6}=1 \mathrm{mB} \mathrm{B}_{6}=32 \mathrm{~g}_{0}^{2} \mathrm{~g}^{2}\left(\mathrm{~g}_{0}^{2}-\mathrm{g}^{2}\right) \cos ^{2} \varphi \sin \varphi \cos ^{2} \varphi_{0}-32 \mathrm{~g}_{0}^{2} \mathrm{~g}^{2}\left(\mathrm{~g}_{0}^{2}-\mathrm{g}^{2}\right) \sin ^{3} \varphi \cos ^{2} \varphi_{0}-32 \mathrm{gg},\left(\mathrm{g}_{0}^{4}-\mathrm{g}^{4}\right) \cos \varphi \sin ^{2} \varphi \cos \varphi_{0} \sin \varphi_{0}-32 \mathrm{~g}_{0}^{2} \mathrm{~g}^{2}\left(\mathrm{~g}_{0}^{2}-\mathrm{g}^{2}\right) \cos$

After some algebraic operations, we arrive at the form:

$$
\begin{aligned}
\mathrm{Q}_{\downarrow} 6= & 32 \mathrm{~g}_{\downarrow} \mathrm{o}^{\uparrow} 2 \mathrm{~g}^{\uparrow} 2\left(\mathrm{~g}_{\downarrow} \mathrm{o}^{\uparrow} 2-\mathrm{g}^{\uparrow} 2\right) \cdot\left(\cos ^{\uparrow} 2 \varphi \sin \varphi \cos ^{\uparrow} 2 \varphi_{\downarrow} \mathrm{O}-\sin ^{\uparrow} 3 \varphi \cos ^{\uparrow} 2 \varphi_{\downarrow} \mathrm{o}-\right. \\
& \cos ^{\uparrow} 2 \varphi_{\downarrow} 0 \sin \varphi \sin ^{\uparrow} 2 \varphi_{\downarrow} 0-0.5 \sin ^{\uparrow} 3 \varphi \sin ^{\uparrow} 2 \varphi_{\downarrow} 0+2 \cos ^{\uparrow} 2 \varphi \sin \varphi \cos ^{\uparrow} 2 \varphi_{\downarrow} 0+0.5 \sin ^{\uparrow} 3
\end{aligned}
$$

and now, the equation for $Q_{6}$ takes the form;

$$
\mathrm{Q}_{6}=0.5\left(\frac{\mathrm{g}_{0}}{\mathrm{~g}}-\frac{\mathrm{g}}{\mathrm{g}_{0}}\right)\left(\sin 3 \varphi \cos ^{2} \varphi_{0}-\cos ^{2} \varphi \sin \varphi \sin ^{2} \varphi_{0}\right)-0.5\left(\frac{\mathrm{g}_{0}^{2}}{\mathrm{~g}^{2}}-\frac{\mathrm{g}^{2}}{\mathrm{~g}_{0}^{2}}\right)\left(\sin 2 \varphi \sin \varphi \sin 2 \varphi_{0}\right)+0.5\left(\frac{\mathrm{g}_{0}^{3}}{\mathrm{~g}^{3}}-\frac{\mathrm{g}^{3}}{\mathrm{~g}_{0}^{3}}\right) \sin ^{3} \varphi \sin ^{2} \varphi_{0}
$$

Taking substitutions:

$$
\mathrm{R}^{\prime}=0.5\left(\frac{\mathrm{g}_{0}}{\mathrm{~g}}-\frac{\mathrm{g}}{\mathrm{g}_{0}}\right), \mathrm{S}^{\prime}=0.5\left(\frac{\mathrm{g}_{0}^{2}}{\mathrm{~g}^{2}}-\frac{\mathrm{g}^{2}}{\mathrm{~g}_{0}^{2}}\right), \mathrm{T}^{\prime}=0.5\left(\frac{\mathrm{g}_{0}^{3}}{\mathrm{~g}^{3}}-\frac{\mathrm{g}^{3}}{\mathrm{~g}_{0}^{3}}\right)
$$

for $\mathrm{Q}_{6}$ finally we have;

$$
\mathrm{Q}_{\mathrm{p}}=\mathrm{R}^{\prime}\left(\sin 3 \varphi \cos ^{2} \varphi_{0}-\cos ^{2} \varphi \sin \varphi \sin ^{2} \varphi_{0}\right)-\mathrm{S}^{\prime}\left(\sin 2 \varphi \sin \varphi \sin \varphi_{0}\right)+\mathrm{T}^{\prime} \sin ^{3} \varphi \sin ^{2} \varphi_{\mathrm{e}}
$$

Determination of denominator $\mathbf{N}_{\mathbf{j}}$ in Eq. 11 for $\mathbf{j}=\mathbf{6}$ : In a similar way as discussed, substituting in successive manner Eq. $25-32$ in Eq. 32 for $\mathrm{N}_{6}$ we have:

$$
\begin{aligned}
\mathrm{N}_{6}= & {\left[4 \mathrm{gg}_{0} \cos \varphi \cos \varphi_{0}+\mathrm{i} 4 \mathrm{gg} \mathrm{g}_{0} \cos \varphi \sin \varphi_{0}-2\left(\mathrm{~g}_{0}^{2}+\mathrm{g}^{2}\right) \sin \varphi \sin \varphi_{0}-2 \mathrm{i}\left(\mathrm{g}_{0}^{2}-\mathrm{g}^{2}\right) \sin \varphi \cos \varphi_{0}\right] } \\
& 16 \mathrm{~g}_{0}^{2} \mathrm{~g}^{2} \cos ^{2} \varphi \cos \varphi_{0}-16 \mathrm{~g}_{0}^{2} \mathrm{~g}^{2} \sin ^{2} \varphi \cos \varphi_{0}-16 \mathrm{~g}_{0} \mathrm{~g}\left(\mathrm{~g}_{0}^{2}+\mathrm{g}^{2}\right) \mathrm{s}
\end{aligned}
$$

We take the real part of 38 that corresponds to parameter $\mathrm{K}$ and imaginary part that corresponds to the parameter L.

\section{Determination of $K_{6}$ and $L_{6}$ parameters:}

or:

$$
\operatorname{ReN}_{\downarrow} 6=64 \mathrm{~g}_{\downarrow} 0^{\uparrow} 3 \mathrm{~g}^{\uparrow} 3\left(\begin{array}{l}
\cos ^{\uparrow} 3 \varphi \cos ^{\uparrow} 2 \varphi_{\downarrow} 0-\cos \varphi \sin ^{\uparrow} 2 \varphi \cos ^{\uparrow} 2 \varphi_{\downarrow} 0-\cos ^{\uparrow} 3 \varphi \sin ^{\uparrow} 2 \varphi_{\downarrow} O+ \\
\cos \varphi \sin ^{\uparrow} 2 \varphi \sin ^{\uparrow} 2 \varphi_{\downarrow} 0-\cos \varphi \sin ^{\uparrow} 2 \varphi \cos ^{\uparrow} 2 \varphi_{\downarrow} 0-\cos \varphi \sin ^{\uparrow} 2 \varphi \cos ^{\uparrow} 2 \varphi_{\downarrow} \mathrm{O}
\end{array}\right)+32 \mathrm{~g}_{\downarrow} \mathrm{o}^{\uparrow} 2
$$

$$
\begin{aligned}
& \operatorname{Re} N_{6}= 64 g_{0}^{3} g^{3}\left(\cos ^{3} \varphi \cos 2 \varphi_{0}-3 \cos \varphi \sin ^{2} \varphi \cos 2 \varphi_{0}-2 \cos \varphi \sin ^{2} \varphi \sin ^{2} \varphi_{0}\right)+ \\
& 32 g_{0}^{2} g^{2}\left(g_{0}^{2}+g^{2}\right)\left[2\left(-3 \cos ^{2} \varphi \sin \varphi+\sin ^{3} \varphi\right)\right]+32 g_{0} g\left(g_{0}^{4}+g^{4}\right) \sin 2 \varphi \sin \varphi \sin ^{2} \varphi_{0} \\
& \\
& {\left[\operatorname{ReN}_{6}=64 g_{0}^{3} g^{3}\left(\cos ^{3} \varphi\right]\left(\cos 2 \varphi_{0}-\sin 2 \varphi \sin \varphi \sin ^{2} \varphi_{0}\right)-\right.} \\
& 32 g_{0}^{2} g^{2}\left(g_{0}^{2}+g^{2}\right) \sin 3 \varphi \sin 2 \varphi_{0}+32 g_{0} g\left(g_{0}^{4}+g^{4}\right) \sin 2 \varphi \sin \varphi \sin ^{2} \varphi_{0}
\end{aligned}
$$


For $\mathrm{K}_{6}$, we obtain:

$$
\mathrm{K}_{6}=\frac{\mathrm{ReN}_{6}}{64 \mathrm{~g}_{0}^{3} \mathrm{~g}^{3}}=\cos 3 \varphi \cos 2 \varphi_{0}-\sin 2 \varphi \sin \varphi \sin ^{2} \varphi_{0}-0.5\left(\frac{\mathrm{g}}{\mathrm{g}}+\frac{\mathrm{g}}{\mathrm{g}_{0}}\right) \sin 3 \varphi \sin 2 \varphi_{0}+0.5\left(\frac{\mathrm{g}_{0}^{2}}{\mathrm{~g}^{2}}+\frac{\mathrm{g}^{2}}{\mathrm{~g}_{0}^{2}}\right) \sin 2 \varphi \sin \varphi \sin ^{2} \varphi_{0}
$$

After substitution, we have:

$$
\mathrm{S}=0.5\left(\frac{\mathrm{g}_{0}^{2}}{\mathrm{~g}^{2}}+\frac{\mathrm{g}^{2}}{\mathrm{~g}_{0}^{2}}\right)
$$

Finally, the parameter $\mathrm{K}_{6}$ is:

$$
\mathrm{K}_{6}=\cos 3 \varphi \cos 2 \varphi_{0}-\sin 2 \varphi \sin \varphi \sin ^{2} \varphi_{0}-\mathrm{R} \sin 3 \varphi \sin 2 \varphi_{0}+\mathrm{S} \sin 2 \varphi \sin \varphi \sin ^{2} \varphi_{0}
$$

The imaginary part of the Eq. 33 that corresponds to the parameter $\mathrm{L}_{6}$ is:

$$
\begin{aligned}
\operatorname{lm} N_{\downarrow} 6= & 64 \mathrm{~g} \mathrm{~J}^{\uparrow} 3 \mathrm{~g}^{\uparrow} 3\left(\cos ^{\uparrow} 3 \varphi_{\downarrow} O \cos \varphi_{\downarrow} O \sin \varphi_{\downarrow} O+\cos ^{\uparrow} 3 \varphi_{\downarrow} O \cos \varphi_{\downarrow} \sin \varphi_{\downarrow} O-\cos \varphi \sin ^{\uparrow} 2 \varphi \cos \varphi_{\downarrow} O \sin \varphi_{\downarrow} O-\right. \\
& \cos \varphi \sin ^{\uparrow} 2 \varphi \cos \varphi_{\downarrow} O \sin \varphi_{\downarrow} O-\cos \varphi \sin ^{\uparrow} 2 \varphi \cos \varphi_{\downarrow} O \sin \varphi_{\downarrow} O+\cos \varphi \sin ^{\uparrow} 2 \varphi \cos \varphi
\end{aligned}
$$

Then, we obtain the equation of the form:

$$
\begin{aligned}
1 m N_{6}= & 64 g_{0}^{3} g^{3}\left(\cos 3 \varphi+\cos \varphi \sin ^{2} \varphi\right) \sin 2 \varphi_{0}+32 g_{0}^{2} g^{2}\left(g_{0}^{2}+g^{2}\right)\left(\sin 3 \varphi \cos 2 \varphi_{0}-\sin ^{3} \varphi \sin ^{2} \varphi_{0}\right)- \\
& 32 g_{0} g\left(g_{0}^{4}+g^{4}\right) \sin 2 \varphi \sin \varphi \sin 2 \varphi_{0}+32\left(g_{0}^{6} g^{6}\right) \sin 3 \varphi \sin ^{2} \varphi_{0}
\end{aligned}
$$

For the parameter $\mathrm{L}_{6}$, we have the equation:

$$
\left.\frac{g_{0}^{3}}{g^{3}}+\frac{g^{3}}{g_{0}^{3}}\right)
$$

After substitution, we have:

$$
\mathrm{T}=0.5\left(\frac{\mathrm{g}_{0}^{3}}{\mathrm{~g}^{3}}+\frac{\mathrm{g}^{3}}{\mathrm{~g}_{0}^{3}}\right)
$$

Finally, we obtain the formula for $\mathrm{L}_{6}$ :

$$
\begin{aligned}
\mathrm{L}_{6}= & \left(\cos 3 \varphi+\cos \varphi \sin ^{2} \varphi\right) \sin 2 \varphi_{0}+ \\
& \mathrm{R}\left(\sin 3 \varphi \cos 2 \varphi_{0}-\sin ^{3} \varphi \sin 2 \varphi_{0}\right)- \\
& \mathrm{S} \sin 2 \varphi \sin \varphi \sin 2 \varphi_{0}+T \sin ^{3} \varphi \sin ^{2} \varphi_{0}
\end{aligned}
$$

Thus, we have determined the coefficients $P, Q, K$ and $\mathrm{L}$ and therefore, the coefficients $\mathrm{r}$ and $\mathrm{t}$ for three parallel plates equally spaced in vacuum, the separation being equal with the thickness of the plates.

Anisotropic plates: In anisotropic materials-crystalline plates, light is propagated always in two different directions with different velocity of propagation.
Therefore, these two waves have different indices of refraction. Nevertheless, there exist directions in which the two waves have the same velocity. This direction is the direction of the optical axis of the crystal. Crystals can have not $>2$ optical axes and are called uniaxial crystals. Light wave in a crystal has the ordinary wave denoted by $\mathrm{O}$ and the extraordinary wave denoted by e.

The ordinary wave has the same velocity $\mathrm{v}_{0}$ in all directions and the end of the velocity vector describe a spherical surface. This wave has the index of refraction $n_{0}$. The extraordinary wave has different velocities in different directions and the end the velocity vector describes an ellypsoidal surface with the optical axis of the crystal as a rotation axis (Zander et al., 1985).

Light waves incident on the uniaxial crystal plates are linearly polarized and oscillate always in certain planes. Therefore, it is necessary to define the principal planes. The principal plane $\mathrm{H}$ is determined by the direction of the propagation of light and the optical axis of the crystal. It is proven that the ordinary wave oscillates perpendicularly to this plane whereas the extraordinary wave oscillates parallel to this plane. These are essential facts that must be taken into consideration in further discussion. If we intent to use mathematical data obtained for isotropic plates which is possible then we have to deal with certain positions of the optical axes in relation to the 
crystal surfaces. In particular, we consider the case when the optical axes of the crystal are parallel to boundary surfaces of the planes of the crystal. These are the most appropriate forms for applications but there are also other cases which we are to discuss.

Optical axes are perpendicular to the surfaces of the plates: In this case, plates are designed appropriately in order that their surfaces be perpendicular to the optical axes and this is valid for all plates we will consider. Here, the optical axis belongs to the incident plane and has the direction of the propagation of light (Moser, 1988).

Therefore, the principal plane is the same as the incident plane. According to the definition, the ordinary wave oscillates perpendicularly to the incident plane whereas the extraordinary wave oscillates parallel to this plane. Here, we will denote the perpendicular componentthe ordinary wave by $o$ and the parallel componentextraordinary wave by e. The phase for the ordinary component of the wave is:

$$
\varphi_{0}=\frac{2 \pi}{\lambda} \mathrm{dn}_{0} \cos \beta_{0}
$$

Whereas, the phase for the extraordinary component of the wave is:

$$
\varphi_{\mathrm{e}}=\frac{2 \pi}{\lambda} \mathrm{dn}_{\mathrm{e}} \cos \beta_{\mathrm{e}}
$$

The phase for the space in vacuum is:

$$
\varphi=\frac{2 \pi}{\lambda} d \cos \alpha
$$

Therefore, for the system of three parallel plates separated in vacuum, we can use the formulas for the parameters $P, Q, K$ and $L$ for isotropic plates 34, 37, 40 and 43 and substitute them for the ordinary wave and extraordinary wave, respectively considering the position of the principal plane $\mathrm{H}$ and the incident plane as defined before. Therefore, for the parameters $\mathrm{P}, \mathrm{Q}, \mathrm{K}$ and $\mathrm{L}$, for normal components, we obtain:

$$
\begin{gathered}
\mathrm{P}_{\mathrm{n}}=0 \\
\mathrm{Q}_{\mathrm{n}}=\mathrm{R}_{0}^{\prime}\left(\sin 3 \varphi_{0} \cos ^{2} \varphi-\cos ^{2} \varphi_{0} \sin \varphi_{0} \sin ^{2} \varphi\right)- \\
\mathrm{S}_{0}^{\prime} \sin 2 \varphi_{0} \sin \varphi_{0} \sin 2 \varphi+\mathrm{T}_{0}^{\prime} \sin ^{3} \varphi_{0} \sin ^{2} \varphi \\
\mathrm{K}_{\mathrm{n}}=\cos 3 \varphi_{0} \cos 2 \varphi-\sin 2 \varphi_{0} \sin \varphi_{0} \sin ^{2} \varphi- \\
\mathrm{R}_{0} \sin 3 \varphi_{0} \sin 2 \varphi+\mathrm{S}_{0} \sin 2 \varphi_{0} \sin \varphi_{0} \sin ^{2} \varphi
\end{gathered}
$$

$$
\begin{aligned}
\mathrm{L}_{\mathrm{n}}= & \left(\cos 3 \varphi_{0}+2 \cos \varphi \sin ^{2} \varphi_{0}\right) \sin 2 \varphi+ \\
& \mathrm{R}_{0}\left(\sin 3 \varphi_{0} \cos 2 \varphi-\sin ^{3} \varphi_{0} \sin ^{2} \varphi\right)- \\
& \mathrm{S}_{0} \sin 2 \varphi_{0} \sin \varphi_{0} \sin 2 \varphi+\mathrm{T}_{0} \sin ^{3} \varphi_{0} \sin _{2} \varphi
\end{aligned}
$$

For parallel components, we have:

$$
\begin{gathered}
\mathrm{P}_{\mathrm{p}}=0 \\
\mathrm{Q}_{\mathrm{p}}=\mathrm{R}_{\mathrm{e}}^{\prime}\left(\sin 3 \varphi_{\mathrm{e}} \cos ^{2} \varphi-\cos ^{2} \varphi_{\mathrm{e}} \sin \varphi_{\mathrm{e}} \sin ^{2} \varphi\right)- \\
\mathrm{S}_{\mathrm{e}}^{\prime} \sin 2 \varphi_{\mathrm{e}} \sin \varphi_{\mathrm{e}} \sin 2 \varphi+\mathrm{T}_{\mathrm{e}}^{\prime} \sin ^{3} \varphi_{\mathrm{e}} \sin ^{2} \varphi \\
\mathrm{K}_{\mathrm{p}}=\cos 3 \varphi_{\mathrm{e}} \cos 2 \varphi-\sin 2 \varphi_{\mathrm{e}} \sin \varphi_{\mathrm{e}} \sin ^{2} \varphi- \\
\mathrm{R}_{\mathrm{e}} \sin 3 \varphi_{\mathrm{e}} \sin 2 \varphi+\mathrm{S}_{\mathrm{e}} \sin 2 \varphi_{\mathrm{e}} \sin \varphi_{\mathrm{e}} \sin ^{2} \varphi \\
\mathrm{L}_{\mathrm{p}}=\left(\cos 3 \varphi_{\mathrm{e}}+2 \cos \varphi_{\mathrm{e}} \sin \varphi_{\mathrm{e}}\right) \sin 2 \varphi+ \\
\mathrm{R}_{\mathrm{e}}\left(\sin 3 \varphi_{\mathrm{e}} \cos 2 \varphi-\sin ^{3} \varphi_{\mathrm{e}} \sin ^{2} \varphi\right)- \\
\mathrm{S}_{\mathrm{e}} \sin 2 \varphi_{\mathrm{e}} \sin \varphi_{\mathrm{e}} \sin 2 \varphi+\mathrm{T}_{\mathrm{e}} \sin ^{3} \varphi_{\mathrm{e}} \sin ^{2} \varphi
\end{gathered}
$$

Optical axes are parallel to the surfaces of the plate and perpendicular to the incident plane: Figure 3 shows a crystal plate with the optical axis OA on the plane of the plate, perpendicular to the incident plane $I I$ which contains the incident beam. This is valid for all three plates. Incident beam covers the incident angle $\beta$ with the normal $\mathrm{n}$.

The principal plane $\mathrm{H}$ contains the incident beam and the optical axis OA. The ordinary beam oscillates perpendicularly to the plane $\mathrm{H}$ and is parallel to the incident plane $\mathrm{D}$ so that this beam is indexed by $\mathrm{O}$ on the position of the index $\mathrm{p}$.

The extraordinary beam e oscillates parallel to the plane $\mathrm{H}$ and is perpendicular to the incident plane and is indexed by $O$ (Zander et al., 1985). Therefore, in the Eq. 44-47, we replace the index o by $\mathrm{e}$ for the normal components and in the Eq. 48-51, we replace the index e by o for the parallel components. Therefore, we have: for the normal component:

$$
\begin{gathered}
\mathrm{P}_{\mathrm{n}}=0 \\
\mathrm{Q}_{\mathrm{n}}=\mathrm{R}^{\prime}\left(\cos 3 \varphi_{\mathrm{e}} \cos ^{2} \varphi-\cos ^{2} \varphi_{\mathrm{e}} \sin \varphi_{\mathrm{e}} \sin ^{2} \varphi\right)- \\
\mathrm{S}_{\mathrm{e}}^{\prime} \sin 2 \varphi_{\mathrm{e}} \sin \varphi_{\mathrm{e}} \cos ^{2} \varphi+\mathrm{T}_{\mathrm{e}} \sin ^{3} \varphi_{\mathrm{e}} \sin ^{2} \varphi \\
\mathrm{K}_{\mathrm{n}}=\cos 3 \varphi_{\mathrm{e}} \cos 2 \varphi-\sin 2 \varphi_{\mathrm{e}} \sin \varphi_{\mathrm{e}} \sin ^{2} \varphi- \\
\mathrm{R}_{\mathrm{e}} \sin 3 \varphi_{\mathrm{e}} \sin 2 \varphi+\mathrm{S}_{\mathrm{e}} \sin 2 \varphi_{\mathrm{e}} \sin \varphi_{\mathrm{e}} \sin ^{2} \varphi
\end{gathered}
$$




$$
\begin{aligned}
& \mathrm{L}_{\mathrm{n}}=\left(\cos 3 \varphi_{\mathrm{e}}+2 \cos \varphi_{\mathrm{e}} \sin ^{2} \varphi_{\mathrm{e}}\right) \sin 2 \varphi+ \\
& \mathrm{R}_{\mathrm{e}}\left(\sin 3 \varphi_{\mathrm{e}} \cos 2 \varphi-\sin ^{3} \varphi_{\mathrm{e}} \sin ^{2} \varphi\right)- \\
& \mathrm{S}_{\mathrm{e}} \sin 2 \varphi_{\mathrm{e}} \sin \varphi_{\mathrm{e}} \sin 2 \varphi+\mathrm{T}_{\mathrm{e}} \sin ^{3} \varphi_{\mathrm{e}} \sin ^{2} \varphi
\end{aligned}
$$

And for the parallel component:

$$
\begin{gathered}
\mathrm{P}_{\mathrm{p}}=0 \\
\mathrm{Q}_{\mathrm{p}}=\mathrm{R}_{0}^{\prime}\left(\sin 3 \varphi_{0} \cos ^{2} \varphi-\cos ^{2} \varphi_{0} \sin \varphi_{0} \sin ^{2} \varphi\right)- \\
\mathrm{S}_{0}^{\prime} \sin 2 \varphi_{\mathrm{e}} \sin \varphi_{0} \sin 2 \varphi+\mathrm{T}_{0}^{\prime} \sin ^{3} \varphi_{0} \sin ^{2} \varphi \\
\mathrm{K}_{\mathrm{p}}=\cos 3 \varphi_{0} \cos 2 \varphi-\sin 2 \varphi_{0} \sin \varphi_{0} \sin ^{2} \varphi- \\
\mathrm{R}_{0} \sin 3 \varphi_{0} \sin 2 \varphi+\mathrm{S}_{0} \sin 2 \varphi_{0} \sin \varphi_{0} \sin ^{2} \varphi \\
\mathrm{L}_{\mathrm{p}}=\left(\cos 3 \varphi_{0}+2 \cos \varphi_{0} \sin ^{2} \varphi_{0}\right) \sin 2 \varphi+ \\
\mathrm{R}_{0}\left(\sin 3 \varphi_{0} \cos 2 \varphi-\sin ^{3} \varphi_{0} \sin ^{2} \varphi\right)- \\
\mathrm{S}_{0} \sin 2 \varphi_{0} \sin \varphi_{0} \sin 2 \varphi+\mathrm{T}_{0} \sin ^{3} \varphi_{0} \sin ^{2} \varphi
\end{gathered}
$$

We see from these equation that they differ from the formulas of the case when the optical axes are perpendicular to the surfaces of the plates, only in switching the indices o and e.

Optical axes are parallel to the surfaces of the plates and have special directions: Let us discuss the case when the principal axis $H$ covers the angle $\gamma$ with the plane $D$ as a

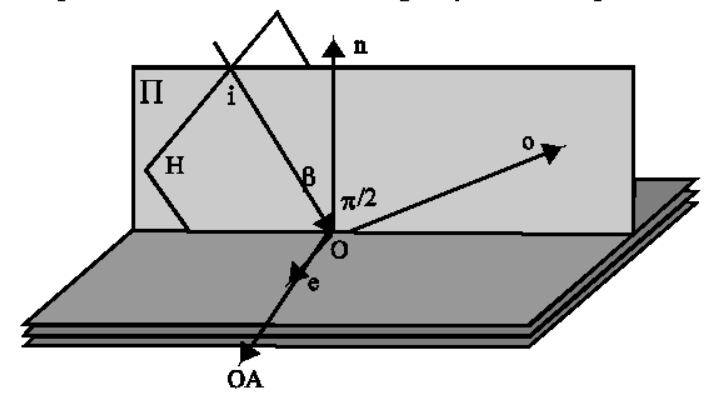

Fig. 3: Crystal plate with optical axis horizontal plane (Fig. 4). The ordinary wave o oscillates perpendicularly to the plane $\mathrm{H}$, whereas the extraordinary wave oscillates parallel to the plane $H$. These waves are inclined to the plane D.

Therefore, the waves o and are decomposed into components that are perpendicular and parallel to the plane D. For the sake of simplicity, we assume that waves have unit amplitudes, and therefore, from Fig. 2, for the ordinary wave o we have:

$$
\mathrm{O}_{\mathrm{n}}=\cos \gamma, \quad \mathrm{O}_{\mathrm{p}}=\sin \gamma
$$

Whereas for the extraordinary wave e, we have the components:

$$
\mathrm{e}_{\mathrm{n}}=\sin \gamma, \quad \mathrm{e}_{\mathrm{p}}=\cos \gamma
$$

From these two equations, we see that in the parallel and perpendicular directions, the ordinary wave o and the extraordinary wave, both contribute which differs from previous cases.

Therefore, for systems composed from three plates, analogously to the previous cases for parameters $\mathrm{P}, \mathrm{Q}, \mathrm{K}$ and $\mathrm{L}$, we will have equations that contain two projections of the components:

$$
\left(\begin{array}{l}
P_{n} \\
P_{p}
\end{array}\right)=0
$$

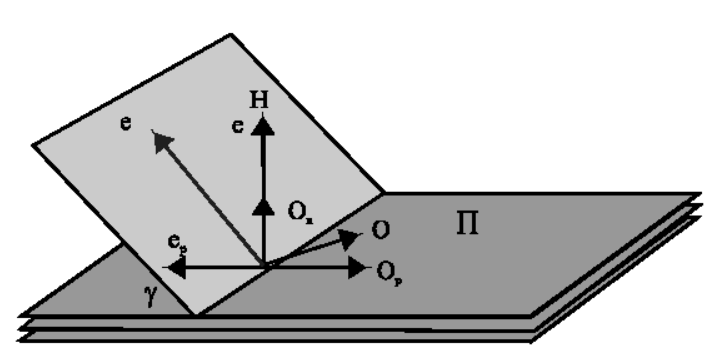

Fig. 4: The plane $\Pi$ as a horizontal plane

$$
\begin{aligned}
& \left(\mathrm{F}\left(\mathrm{Q}_{\downarrow} \mathrm{n} @ \mathrm{Q} \mathrm{Q}_{\downarrow} \mathrm{p}\right)\right)=\left[\mathrm{R}_{\downarrow} \mathrm{o}^{\uparrow}\left(\sin 3 \varphi_{\downarrow} \mathrm{o} \cos ^{\uparrow} 2 \varphi-\cos ^{\uparrow} 2 \varphi_{\downarrow} \mathrm{o} \sin \varphi_{\downarrow} \mathrm{o} \sin ^{\uparrow} 2 \varphi\right)-\mathrm{S}_{\downarrow} \mathrm{o}^{\uparrow} \sin 2 \varphi_{\downarrow} \mathrm{osin} \varphi_{\downarrow} \mathrm{O} \sin 2 \varphi+\mathrm{T}_{\downarrow} \mathrm{o}^{\uparrow}\right. \text { ('t2ntes equally spaced in vacc" } \\
& \left(F\left(Q_{\downarrow} n @ K_{\downarrow} p\right)\right)=\left(\cos 3 \varphi_{\downarrow} O \cos 2 \varphi-\sin 2 \varphi_{\downarrow} O \sin \varphi_{\downarrow} O \sin ^{\uparrow} 2 \varphi-R_{\downarrow} O \sin 3 \varphi_{\downarrow} O \sin 2 \varphi+S_{\downarrow} O \sin 2 \varphi_{\downarrow} O \sin \varphi_{\downarrow} O \sin ^{\uparrow} 2 \varphi\right) \\
& (F(\cos \gamma @ \sin \gamma))+\left(\cos 3 \varphi_{\downarrow} \mathrm{e} \cos 2 \varphi-\sin 2 \varphi_{\downarrow} \mathrm{e} \sin \varphi_{\downarrow} \mathrm{e} \sin ^{\uparrow} 2 \varphi-\mathrm{R}_{\downarrow} \mathrm{es}\right. \\
& \left(\mathrm{F}\left(\mathrm{L}_{\downarrow} \mathrm{n} @ \mathrm{~L}_{\downarrow} \mathrm{p}\right)\right)=\left[\begin{array}{l}
\left(\cos 3 \varphi_{\downarrow} \mathrm{o}+2 \cos \varphi_{\downarrow} \mathrm{o} \sin ^{\uparrow} 2 \varphi_{\downarrow} \mathrm{o}\right) \sin 2 \varphi+\mathrm{R}_{\downarrow} \mathrm{o}\left(\sin 3 \varphi_{\downarrow} \mathrm{o} \cos 2 \varphi-\sin ^{\uparrow} 3 \varphi_{\downarrow} \mathrm{o} \sin ^{\uparrow} 2 \varphi\right)-\mathrm{S}_{\downarrow} \mathrm{o} \sin ^{\prime} 2 \varphi_{\downarrow} \mathrm{osin} \varphi_{\downarrow} \mathrm{osin} 2 \varphi+ \\
\mathrm{T}_{\downarrow} \mathrm{o} \sin ^{\uparrow} 3 \varphi_{\downarrow} \mathrm{o} \sin ^{\uparrow} 2 \varphi(\mathrm{F}(\cos \gamma @ \sin \gamma))+\left[\left(\cos 3 \varphi_{\downarrow} \mathrm{e}+2 \cos \right)\right]
\end{array}\right]
\end{aligned}
$$




\section{RESULTS AND DISCUSSION}

The accuracy of the Eq. 34, 37, 40 and 43 is verified if we consider all three plates as a single plate for $\varphi_{0}=0$ and from Eq. 34, 37, 40 and 43 we obtain the equations of the form:

$$
\begin{array}{ll}
P_{6}=0 & Q_{6}=R^{\prime} \sin 3 \varphi \\
P_{6}=\sin 3 \varphi & L_{6}=R \sin 3 \varphi
\end{array}
$$

From Eq. 61, expressions for $\mathrm{P}_{6}, \mathrm{Q}_{6}, \mathrm{~K}_{6}$ and $\mathrm{L}_{6}$ are proved to be accurate. These are the same as in the case of e single plate Eq. 24 with a tripled thickness d. Also for the case of anisotropic plates, Eq. 44-51 turn to be the same as for a single plate with thickness $3 \mathrm{~d}$. In this case, for $\varphi=0$ we have:

$$
\begin{array}{ll}
\mathrm{P}_{\mathrm{n}}=0 & \mathrm{Q}_{\mathrm{n}}=\mathrm{R}_{0}^{\prime} \sin 3 \varphi_{0} \\
\mathrm{~K}_{\mathrm{n}}=\cos 3 \varphi_{0} & \mathrm{~L}_{\mathrm{n}}=\mathrm{R}^{\prime} \sin 3 \varphi_{0}
\end{array}
$$

and;

$$
\begin{array}{cl}
\mathrm{P}_{\mathrm{p}}=0 & \mathrm{Q}_{\mathrm{p}}=\mathrm{R}_{\mathrm{e}}^{\prime} \sin 3 \varphi_{0} \\
\mathrm{~K}_{\mathrm{p}}=\cos 3 \varphi_{0} & \mathrm{~L}_{\mathrm{p}}=\mathrm{R}^{\prime} \sin 3 \varphi_{0}
\end{array}
$$

Moreover, coefficients $\mathrm{r}$ and $\mathrm{t}$ are complex quantities, showing that reflected and transmitted light has an elliptical polarization. Values for R', S' and T' are different for parallel and normal component. This is also valid for values of $R, S$ and $T$. Therefore, quantities $Q, K$ and $L$ are determined for normal and parallel component.

\section{CONCLUSION}

Formulas obtained for $\mathrm{P}, \mathrm{Q}, \mathrm{K}$ and $\mathrm{L}$ for isotropic plates can be transformed for the anisotropic plates as well but specifying the positions of the optical axis, the principal plane and the incident plane. As far as, these parameters were determined, the determination of the azimuthal angle $\psi$ of the reflected and transmitted light as a function of the azimuth of the incident light $\psi_{0}$ and the ellypticity $v$ is made possible. Light has a high degree of coherence.

\section{REFERENCES}

Born, M. and E. Wolf, 1999. Principles of Optics. 7th Edn., Cambridge University Press, England, pp: 22.

Moser, J., 1985. The polarization of monochromatic light after reflection on a plan parallel plate. Physics, 18: 157-159.

Moser, J., 1988. The polarization state of light in plan parallel uniaxial crystalline plate. Physics, 39: 49-62.

Moser, J., L. Janicijevic and M. Janoska, 1987. The polarization state of light transmitted by a plate and liquid layer. Optics, 67: 27-31.

Zander, K., H. Melle and J. Moser, 1983. The polarization state of coherent light in anisotropic and isotropic plan parallel plates. RTB-Berichet Optics, 14: 1-40.

Zander, K., J. Moser and H. Melle, 1985. Change of polarization of linearly polarized coherent light transmitted through plane-parallel anisotropic plates. Optics, 70: 6-13. 